\title{
CONVOLUTION PRESERVES PARTIAL SYNCHRONICITY OF LOG-CONCAVE SEQUENCES
}

\author{
Han Hu, David G. L. Wang, Feng Zhao and Tongyuan ZhaO
}

\begin{abstract}
In a recent proof of the log-concavity of genus polynomials of some families of graphs, Gross et al. defined the weak synchronicity relation between log-concave sequences, and conjectured that the convolution operation by any log-concave sequence preserves weak synchronicity. In this paper we disprove it by providing a counterexample. Furthermore, we introduce the so-called partial synchronicity relation between log-concave sequences, which is proved to be (i) weaker than synchronicity, (ii) stronger than weak synchronicity, and (iii) preserved by the convolution operation.
\end{abstract}

Mathematics subject classification (2010): 05A20, 26D05.

Keywords and phrases: Log-concavity, synchronicity, sequence convolution, combinatorial inequality.

\section{REFERENCES}

[1] L. W. Beineke, R. J. Wilson, J. L. Gross, And T. W. Tucker, Topics in Topological Graph Theory, Cambridge Univ. Press (2009).

[2] J. BorCEA, P. BRÄNDÉN, AND T. M. LigGeTt, Negative dependence and the geometry of polynomials, J. Amer. Math. Soc. 22 (2) (2009), 521-567.

[3] F. BRenti, Unimodal, log-concave and Pólya frequency sequences in combinatorics, Mem. Amer. Math. Soc., Vol. 81, no. 413, American Mathematical Society, Providence, RI (1989).

[4] F. BRENTI, Log-concave and unimodal sequences in algebra, combinatorics, and geometry: An update, Contemp. Math., Vol. 178, Jerusalem combinatorics '93, eds. H. Barcelo and G. Kalai, American Mathematical Society, Providence, RI (1994), 71-89.

[5] J. Gross, T. Mansour, T. Tucker, And D. G. L. WANG, Log-concavity of combinations of sequences and applications to genus distributions, SIAM J. Discrete Math. 29 (2) (2015), 1002-1029.

[6] J. L. Gross, D. P. RobBins, And T. W. Tucker, Genus distributions for bouquets of circles, J. Combin. Theory Ser. B 47 (3) (1989), 292-306.

[7] J. L. Gross And T. W. TuCKer, Topological Graph Theory, Dover Publications (2012), reprint.

[8] T. M. LiggetT, Ultra logconcave sequences and negative dependence, J. Combin. Theory Ser. A 79 (2) (1997), 315-325.

[9] K. V. Menon, On the convolution of logarithmically concave sequences, Proc. Amer. Math. Soc. 23 (2) (1969), 439-441.

[10] R. P. STANLEY, Log-concave and unimodal sequences in algebra, combinatorics, and geometry, Ann. New York Acad. Sci. 576 (1) (1989), 500-534. 